\title{
Global Volumetric Estimation of Disease Burden in Multiple Sclerosis Based on Magnetization Transfer Imaging
}

Mark A. van Buchem, Jayaram K. Udupa, Joseph C. McGowan, Yukio Miki, Fenna H. Heyning, Marie-Paule Boncoeur-Martel, Dennis L. Kolson, Marcy Polansky, and Robert I. Grossman

\begin{abstract}
Summary: We report a semiautomated postprocessing method based on magnetization transfer MR imaging that can quantify the extent of global disease in patients with multiple sclerosis. The technique combines segmentation and quantitative analysis of imaging data reflecting the structural integrity of white matter. Applications of this technique may include assessment of disease progress and of the efficacy of experimental therapeutic intervention. The height of the histogram peak corresponding to white matter was found to be lowered in patients with multiple sclerosis and the overall distribution of magnetization transfer ratios was shifted to lower values.
\end{abstract}

Index terms: Magnetic resonance, magnetization transfer; Magnetic resonance, tissue characterization; Sclerosis, multiple

The recently introduced technique known as magnetization transfer (MT) imaging may be more sensitive to the presence of multiple sclerosis (MS) than are conventional magnetic resonance (MR) imaging techniques. Animal experiments in a model of wallerian degeneration with histopathologic confirmation have suggested that MT imaging is sensitive to structural changes in white matter that are not apparent by conventional imaging (1). In MS, abnormalities have been detected not only in plaques but also in areas of white matter that appeared normal on conventional MR images (2-4). These abnormalities in normal-appearing white matter have been attributed to the microscopic parenchymal disease that has been found in histologic studies.

Estimation of the extent (or burden, as assessed radiologically) of disease in MS is desirable in order to study the natural course of the disease and to evaluate the effects of treatment.
Little correspondence has been established between clinical gradations of the disease and extent of disease apparent at MR imaging: some patients with markedly abnormal MR studies are clinically asymptomatic while others with only minimal disease apparent on MR images are profoundly impacted by the disease. These discrepant findings may be explained by the observation that clinical assessment of disease extent is heavily weighted toward spinal cord and motor tract involvement. It is clear that disease burden in a clinical sense may be quite different from, and not associated with, disease extent as seen on MR images. In the case of MS, the lack of clinical symptoms may actually mask the progress of disease.

Recently, we showed that analysis of threedimensional MT data from a slab of unsegmented cranial tissue by means of MT ratio histograms could be used to estimate the total extent of MS in that slab (5). The present work combines segmentation of total brain parenchyma with generation of an MT ratio histogram, resulting in a more comprehensive assessment of disease burden.

\section{Technique}

MR imaging was performed on a Signa (General Electric Medical Systems, Milwaukee, Wis) 1.5-T unit equipped with a quadrature transmit/receive head coil. MT imaging was performed with a 3-D gradient-echo pulse sequence with parameters of $106 / 5 / 1$ (repetition time/ echo time/excitations) and a $12^{\circ}$ flip angle. A matrix of $256 \times 128$ was used for a total of 28 sections with a section thickness of $5 \mathrm{~mm}$. The field of view was $22 \mathrm{~cm}$.

Received May 21, 1996; accepted after revision October 16.

Supported in part by grants NS29029, M01-RR00040, R55-34353, and RO1 CA56071 from the National Institutes of Health.

From the Departments of Radiology (M.A.V.B., J.K.U., J.C.M.G., Y.M., F.H.H., M-P.B-M., R.I.G.) and Neurology (D.L.K.), Hospital of the University of Pennsylvania, Philadelphia; the Department of Radiology (M.A.V.B.), Leiden (the Netherlands) University Hospital; and the Division of Biometrics, Hahnemann University, Philadelphia (M.P.).

Address reprint requests to Joseph C. McGowan, Department of Radiology, Hospital of the University of Pennsylvania, 3400 Spruce St, Philadelphia, PA 19104. 
Two consecutive sets of axial images were obtained: the first set with the radio-frequency $(\mathrm{RF})$ saturation pulse on and the second with the saturation pulse off. A sinc-shaped $\mathrm{RF}$ saturation pulse with an average field intensity equal to $3.67 \times 10^{-6} \mathrm{~T}$ was applied at a frequency $2 \mathrm{kHz}$ below water resonance (6). The approximate acquisition time for each set of images was 7.3 minutes. Image postprocessing was performed on a Sun Sparc 20 workstation, with the amount of MT quantified as described previously (3). All image processing operations were carried out by using 3DVIEWNIX software (J. K. Udupa, D. Odhner, S. Samarasekera, "3DVIEWNIX: An Open, Transportable, Multidimensional, Multimodality, Multiparametric Imaging Software System," presented at the annual meeting of the Society of Photo-Optical Instrumentation Engineers, Newport Beach, Calif, February 1994). The following steps were executed for each patient's study.

1. Image data were accessed and converted to the format of 3DVIEWNIX.

2. MT images corresponding to the RF saturation pulse on and off were split into two 3-D volumetric images, which we refer to as $I_{s}$ and $I_{o}$, respectively.

3. An operator outlined selected regions occupied by cerebrospinal fluid (CSF) in one section in $I_{s}$.

4. $\mathrm{I}_{\mathrm{s}}$ and $\mathrm{I}_{\mathrm{o}}$ were thresholded at $0.35 \times \mathrm{SI}_{\mathrm{s}}$ and $0.7 \times \mathrm{SI}_{\mathrm{o}}$, respectively, to generate binary 3-D images $\mathrm{I}_{\mathrm{sb}}$ and $\mathrm{I}_{\mathrm{ob}}$. The constraints in these parameters were arrived at empirically. A logical AND operation was done between $I_{s b}$ and $\mathrm{I}_{\mathrm{ob}}$ to generate a new 3-D binary image, $\mathrm{I}_{\mathrm{b}}$. That is, a voxel $v$ in $\mathrm{I}_{\mathrm{b}}$ was assigned a value of 1 if the corresponding voxels in $\mathrm{I}_{\mathrm{sb}}$ and $\mathrm{I}_{\mathrm{ob}}$ both had a value of 1 ; otherwise $v$ was assigned a value of 0 .

5. A mask was created for brain parenchyma based on a standard operation to eliminate structures that are peripherally connected to an object of interest (7). First, $I_{b}$ was dilated by one voxel (any 0 -value voxels adjacent to 1 -value voxels were converted to 1 -value voxels), yielding binary image $\mathrm{I}_{\mathrm{b} 1}$. Then $\mathrm{I}_{\mathrm{b} 1}$ was eroded (opposite of dilated) by one voxel. Erosion was applied seven times successively to the resulting images, eventually yielding binary image $\mathrm{I}_{\mathrm{b} 2}$. Subsequently, $\mathrm{I}_{\mathrm{b} 2}$ was dilated six times to yield binary image $\mathrm{I}_{\mathrm{b} 3}$.

6. A logical AND operation was carried out between $\mathrm{I}_{\mathrm{b}}$ (of step 4) and $\mathrm{I}_{\mathrm{b} 3}$ (of step 5), producing binary image $\mathrm{I}_{\mathrm{b} 4}$. Three-dimensionally connected components were tracked in $\mathrm{I}_{\mathrm{b} 4}$ (8). Components that contained less than 1000 voxels were deleted. The resulting 3-D binary image was denoted $\mathrm{I}_{\mathrm{b} 5}$.

7. Binary sections of $\mathrm{I}_{\mathrm{b} 5}$ overlaid on the corresponding sections of $I_{s} / I_{o}$ were reviewed by an operator to remove remaining fragments of extraaxial structures. Upon correction, this step produced a 3-D binary image, $\mathrm{I}_{\mathrm{b} 6}$, that represented the intraaxial compartment of the study under consideration.

8. Image $\mathrm{I}_{\mathrm{b} 6}$ contained both brain parenchyma and CSF. Separation of brain parenchyma from CSF was based on the different MT ratios between the two. Separation was obtained by using an MT ratio threshold equal to $5 \%$ for the voxels in $\mathrm{I}_{\mathrm{b} 6}$ to consider for further analysis. For these voxels in the region indicated by $\mathrm{I}_{\mathrm{b} 6}$, a histogram of the MT ratios was computed from $I_{s}$ and $I_{o}$. Histograms were normalized by dividing the individual bins by the total number of voxels, $\mathrm{V}_{\mathrm{t}}$, with an MT ratio that was larger than $5 \%$ in $\mathrm{I}_{\mathrm{b} 6}$. The following parameters were computed from this histogram: the peak height, $\mathrm{H}_{\mathrm{p}}$, of the histogram; the MT ratio value corresponding to the peak $M T R_{p}$; the mean MT ratio value, $M T R_{m}$; and the MT ratios $M T R_{25}, M T R_{50}$, and $\mathrm{MTR}_{75}$, corresponding to the 25th, 50th, and 75th percentiles of the histogram, which indicate the MT value at which the respective integral of the histogram is $25 \%$, $50 \%$, and $75 \%$ of the total area.

The study group consisted of 11 patients, including eight women and three men (mean age, 44 years) with clinically diagnosed MS as defined by the Poser criteria (9). The selected group was diverse as to the clinical type of MS they had; at the time of examination, six patients had relapsing-remitting MS and five patients had chronic progressive MS. The control group consisted of 11 healthy volunteers, including six women and five men with a mean age of 31 years.

Steps 3 and 7 of the image postprocessing method involved subjective evaluation of images by the observers to identify CSF and to outline extraaxial tissue. The effect of these interventions on the reproducibility of the parameters of the MT ratio histogram was evaluated as follows. To assess interobserver variability, four investigators performed steps 3 through 8 in 10 studies, seven obtained in MS patients (selected to represent a variety with respect to the morphology of the MT ratio histograms) and three obtained in control subjects. To test intraobserver variability using the parameter $\mathrm{H}_{\mathrm{p}}$, the same MT ratio studies were postprocessed a second time by the same observers, with an interval between the first and second evaluations of at least 24 hours. Interobserver and intraobserver correlations were both in excess of $99 \%$ by intraclass correlation test.

The Mann-Whitney test for independent groups was used to compare the values of $H_{p}, M T R_{p}, M T R_{25}, M T R_{50}$, $M T R_{75}$, and $V_{t}$ in the study and control groups. Since the mean age of the subjects in the two groups differed, the Spearman test was performed to correlate ages and values of $\mathrm{H}_{\mathrm{p}}$ for the study group in order to evaluate the potential influence of age on histograms of MT ratios. In addition, an analysis of covariance with age as a covariant was performed to compensate for the influence of age on the values of $H_{p}$ for the patients and control subjects. The presence of correlations between $\mathrm{H}_{\mathrm{p}}$ and other histogram parameters was evaluated by means of Spearman's test. In all statistical analyses, a $P$ value of less than .05 was assumed to represent a significant difference.

\section{Discussion}

In both MS patients and healthy control subjects, the histograms of MT ratios were characterized by a single and relatively high peak (Fig $1)$. The position of this peak in patients did not 


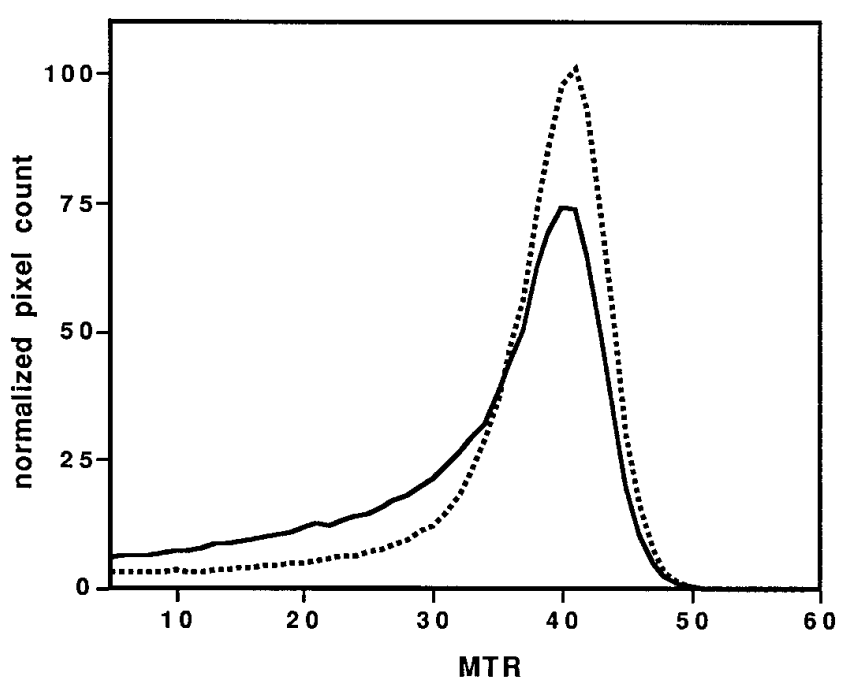

Fig 1. Representative whole brain MT ratio histograms of a healthy subject (dotted line) and a patient with MS (solid line).

differ from that in control subjects ( $P$ not significant). Nevertheless, the histograms of the two groups showed differences. The average normalized peak height, $\mathrm{H}_{\mathrm{p}}$, of the MT ratio histogram in patients was lower than that of control subjects (73.6 \pm 11.5 in patients; $92.4 \pm 7.3$ in control subjects; mean $\pm \mathrm{SD}, P<.0005)$. The range of peak heights was larger in patients (range, 47.9 to 88.4 ) than in control subjects (range, 78.9 to 100 ). In addition, the MT ratio histograms of the patients showed differences in shape as compared with those of the control subjects (Fig 1), characterized by a shift of the distribution to lower MT ratios. This shift was demonstrated by lower MTR $_{25}(P<.05)$ and MTR $_{50}(P<.05)$ values and similar MTR $_{75}(P$ not significant) values relative to those of control subjects. The $M T R_{\mathrm{m}}$ values were lower in the patients than in the control group $(P<.05)$, also reflecting the increased number of voxels with decreased MT ratios in patients. No differences in brain volume between groups were found. In patients, $\mathrm{H}_{\mathrm{p}}$ was correlated with $M \mathrm{TR}_{25}$ $(r=.68 ; P<.05)$ and $M_{T R}(r=.66 ; P<.05)$. In addition, a significant positive correlation was found between $\mathrm{H}_{\mathrm{p}}$ and $\mathrm{MTR}_{\mathrm{m}}(r=.65 ; P<.05)$. $M_{T T}, M T R_{p}$, and $V_{t}$ did not demonstrate correlation with $\mathrm{H}_{\mathrm{p}}$ ( $P$ not significant).

In patients, no correlation was found between $\mathrm{H}_{\mathrm{p}}$ and age ( $P$ not significant); therefore, the difference in $\mathrm{H}_{p}$ between patients and control subjects is unlikely to be explained by the difference in age between these groups. This conclusion is further supported by an analysis of covariance, demonstrating that, with age as a covariant, the difference in $\mathrm{H}_{\mathrm{p}}$ between patients and control subjects was significant $(P<.005)$. No significant variance was added to the parameter $\mathrm{H}_{\mathrm{p}}$ by the operator-dependent postprocessing steps.

In the control group, the height and relative narrowness of the MT ratio histogram peak indicate that most normal brain tissues exhibit MT ratios that lie within a narrow range, in contrast to results in MS patients. The lowering of the peak height of the histogram in patients is the result of an increase in the number of voxels with MT ratios no longer in the range of normal white matter. The possible influence of MS-related brain atrophy on the differences in the histograms between the two populations is excluded by the observation that no differences in brain volume between the two populations exist. The MS patient population thus differs from the healthy subjects in that the volume of brain tissue with a normal range of MT ratios has decreased owing to an increased brain volume with low MT ratios. We presume that this increase is caused by histologic phenomena, such as demyelination, edema, and gliosis, which are known to give rise to a decrease in MT ratios in brain parenchyma. Included in the measured effect is the presence of plaques and the presence of areas of abnormal MT ratio within normal-appearing tissue. The histogram measurement is naturally weighted by both the extent and severity of disease, reflected in the magnitude of decrease in the MT ratio in a region as well as in the size of the affected region.

Previous MT studies in MS have been based on measurements of the MT effect in small twodimensional regions of interest and are reflected by the mean MT ratio value in such regions (2-4, 10-13). This method has proved to be useful for the detection of disease in the brain locally, but to quantitate the extent of visible and occult MS disease in the whole brain or central nervous system, a global approach, such as that proposed in this work, is desirable. Our findings suggest that the peak height of the MT ratio histogram reflects the amount of remaining normal brain parenchyma, as defined by the MT ratio, and might be expected to be inversely related to the amount of parenchyma affected by the disease.

Both manual and computerized counting and measuring of lesion load have been used to assess MS disease burden. Compared with these methods, analysis of MT ratio histograms 
may offer several advantages. First, the technique is sensitive to occult disease, and thus sensitive to abnormal MT ratios, which have been found in plaques as well as in normalappearing white matter. Second, as compared with methods based on manual lesion counts, analysis of MT ratio histograms has the advantage of being objective and highly reproducible (14) (J. Grimaud, M. Lai, J. Thorpe, et al. "Evaluation of a Computer-Assisted Quantification of MS Lesions in Cranial MR Imaging," presented at the Society of Magnetic Resonance Third Scientific Meeting and the European Society for Magnetic Resonance in Medicine and Biology Twelfth Annual Meeting, Nice, France, August 1995). Third, compared with existing automated methods for counting and measuring MS lesions, analysis of MT ratio histograms involves no decisions regarding signal intensity thresholds and may be more objective. Fourth, the time required to obtain a whole-brain MT ratio histogram and its parameters (10 to 15 minutes) is shorter than the time reported for other methods.

In summary, we have undertaken the quantification of white-matter changes in MS based on a segmented, whole-brain analysis of MT images. The presence of visible as well as occult disease was reflected in lowered histogram peak height and a shift in the distribution of MT ratios toward lower values. The technique can characterize disease burden and perhaps assess the efficacy of experimental therapeutic intervention.

\section{Acknowledgment}

We thank Anne Menkens for critically reviewing the manuscript.

\section{References}

1. Lexa FJ, Grossman RI, Rosenquist AC. MR of wallerian degeneration in the feline visual system: characterization by magnetization transfer rate with histopathologic correlation. AJNR Am J Neuroradiol 1994;15:201-212

2. Loevner LA, Grossman RI, Cohen JA, Lexa FJ, Kessler D, Kolson DL. Microscopic disease in normal-appearing white matter on conventional MR images in patients with multiple sclerosis: assessment with magnetization-transfer measurements. Radiology 1995; 196:511-515

3. Dousset V, Grossman RI, Ramer KN, et al. Experimental allergic encephalomyelitis and multiple sclerosis: lesion characterization with magnetization transfer imaging. Radiology 1992;182:483491 (and Erratum, in Radiology 1992;183:878)

4. Hiehle JFJ, Lenkinski RE, Grossman RI, et al. Correlation of spectroscopy and magnetization transfer imaging in the evaluation of demyelinating lesions and normal appearing white matter in multiple sclerosis. Magn Reson Med 1994;32:285-293

5. van Buchem MA, McGowan JC, Kolson DL, Polansky M, Grossman RI. Quantitative volumetric magnetization transfer analysis in multiple sclerosis: estimation of macroscopic and microscopic disease burden. Magn Reson Med 1996;36:632-636

6. McGowan JC, Schnall MD, Leigh JS. Magnetization transfer imaging with pulsed off-resonance saturation: contrast variation with saturation duty cycle. J Magn Reson Imaging 1994;4:79-82

7. Serra J. Image Analysis and Mathematical Morphology. London, England: Academic Press; 1982

8. Udupa J, Ajjanagaddle V. Boundary and object labeling in threedimensional images. Comput Vision Graph Image Proc 1990;51: 355-369

9. Poser CM, Paty DW, Scheinberg L, et al. New diagnostic criteria for multiple sclerosis: guidelines for research protocols. Ann Neurol 1983;13:227-231

10. Hiehle JF Jr, Grossman RI, Ramer KN, Gonzalez-Scarano F, Cohen JA. Magnetization transfer effects in MR-detected multiple sclerosis lesions: comparison with gadolinium-enhanced spinecho images and nonenhanced T1-weighted images. AJNR Am J Neuroradiol 1995;16:69-77

11. Loevner LA, Grossman RI, McGowan JC, Ramer KN and Cohen JA. Characterization of multiple sclerosis plaques with T1weighted MR and quantitative magnetization transfer. AJNR Am J Neuroradiol 1995;16:1473-1479

12. Dousset V, Brochet B, Vital A, et al. Lysolecithin-induced demyelination in primates: preliminary in vivo study with MR and magnetization transfer. AJNR Am J Neuroradiol 1995;16:225231

13. Filippi M, Campi A, Dousset V, et al. A magnetization transfer imaging study of normal-appearing white matter in multiple sclerosis. Neurology 1995;45:478-482

14. Wicks DA, Tofts PS, Miller DH, et al. Volume measurement of multiple sclerosis lesions with magnetic resonance images: a preliminary study. Neuroradiology 1992;34:475-479 